\title{
BMJ Open Risk factors of diabetic retinopathy and sight-threatening diabetic retinopathy: a cross-sectional study of 13473 patients with type 2 diabetes mellitus in mainland China
}

\author{
Yan Liu, ${ }^{1}$ Jiarui Yang, ${ }^{1}$ Liyuan Tao, ${ }^{2}$ Huibin Lv, ${ }^{1}$ Xiaodan Jiang, ${ }^{1}$ Mingzhou Zhang, ${ }^{1}$ \\ Xuemin $\mathrm{Li}^{1}$
}

To cite: Liu Y, Yang J,

Tao L, et al. Risk factors of diabetic retinopathy and sight-threatening diabetic retinopathy: a cross-sectional study of 13473 patients with type 2 diabetes mellitus in mainland China. BMJ Open 2017;7:e016280. doi:10.1136/ bmjopen-2017-016280

\section{- Prepublication history for} this paper is available online. To view these files please visit the journal online (http://dx.doi. org/10.1136/bmjopen-2017016280).

YL and JY contributed equally.

Received 8 February 2017

Revised 1 August 2017

Accepted 9 August 2017

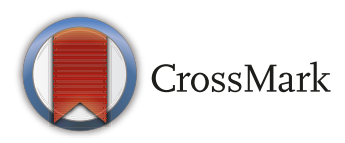

${ }^{1}$ Department of Ophthalmology, Peking University Third Hospital, Beijing, China

${ }^{2}$ Research Center of Clinical Epidemiology, Peking University Third Hospital, Beijing, China

Correspondence to

Dr Xuemin Li;

Ixmlxm66@sina.com

\section{ABSTRACT}

Objective To explore the risk factors of diabetic retinopathy (DR) and sight-threatening diabetic retinopathy (STDR) among Chinese patients with diabetes.

Design, setting and participants A cross-sectional investigation was performed in eight screening clinics in six provinces across mainland China. Information about the risk factors was recorded in screening clinics. Some risk factors (sex, age, diagnosis age, diabetes duration, systolic blood pressure (SBP), diastolic blood pressure, fasting blood glucose (FBG) and glycosylated haemoglobin $(\mathrm{HbA1C}))$ were recorded in all eight clinics, while others were collected only in a subset of the clinics. The relationships between the risk factors and DR and between the risk factors and STDR were explored for the eight factors mentioned above and for all factors studied.

Main outcomes and measures Risk factors of DR and STDR were assessed, and a nomogram of the results was produced.

Results Younger age, longer diabetes duration, higher $\mathrm{SBP}$, higher $\mathrm{FBG}$ and higher $\mathrm{HbA1c}$ were found to be independent risk factors for both DR and STDR in the eight-factor analyses. In the all-factor analysis, younger age, longer diabetes duration, higher SBP, oral medicine use and insulin use were independent risk factors for both DR and STDR; higher postprandial blood glucose (PBG), HbA1c, triglyceride andlow-density lipoprotein were independent risk factors for DR only, and higher FBG was a risk factor for STDR only.

Conclusions In this cross-sectional investigation, several risk factors were found for DR and STDR. Notably, FBG, $\mathrm{PBG}$ and $\mathrm{HbA1C}$ were all risk factors for DR or STDR, suggesting that stricter blood glucose control in clinical practice is required.

\section{INTRODUCTION}

Diabetes mellitus (DM) is a metabolic syndrome with an increasing prevalence and high mortality rate. ${ }^{1}$ Diabetic retinopathy (DR) is a common ocular complication of $\mathrm{DM}$ and is considered to be one of the leading causes of vision loss and vision impairment

\section{Strengths and limitations of this study}

- This is a cross-sectional population-based (13473 subjects) investigation of the risk factors for diabetic retinopathy (DR).

- The study was performed in eight hospitals from six different provinces in mainland China, and participants were from hospitals and communities that included rural and urban regions.

- We separately analysed the risk factors for DR and sight-threatening diabetic retinopathy (STDR), both of which have implications for clinical practice.

- Owing to the multicentre design, some information was not comprehensively collected, which resulted in an imperfect risk factor analysis.

- The sampling method of this study was not stratified, which might result in a lack of representativeness.

in adults. ${ }^{2}$ With the progression of DR, the quality of life of patients decreases, and the financial burden on society increases, both in the DR screening and treatment groups. ${ }^{34}$

DR has been considered to be correlated with many other diabetes-related complications, such as nephropathy, peripheral neuropathy, low bone density and cardiovascular events, all of which lower the quality of life and produce a high rate of mortality. ${ }^{5-8}$ Therefore, early diagnosis and proper management of DR would be of great significance.

Many epidemiological studies on DR, either cross-sectional studies ${ }^{9-18}$ or cohort studies, ${ }^{19-28}$ have been conducted worldwide, exploring the risk factors that were associated with the disease and aiming at the prevention and management of this disease. Older female patients with a longer disease duration were known to be at greater risk for DR and DR progression. Furthermore, having 
renal complications of diabetes, poor glycaemic control, high lipid levels or hypertension have also already been reported to be risk factors of DR, with an impact on DR progression. ${ }^{9-26}$ These factors have been evaluated using the Early Treatment of Diabetic Retinopathy Study classification. ${ }^{27}$ Of these reported risk factors, the duration of diabetes, hyperglycaemia and hypertension were considered to be the most important risk factors for progression of vision loss. ${ }^{29}$ However, DR and the risk factors of DR have typically gained little attention, and the compliance with eye screenings is often poor. ${ }^{30}$ More studies and an improved awareness of the risk factors are therefore required.

In China, a few DR screening studies have been conducted, but most have been completed only in a limited area. ${ }^{21} 2528$ Therefore, a cross-sectional investigation in six provinces (including the northern and southern parts of China) was conducted. The prevalence of DR and its basic epidemiological characteristics have been reported in a previously published article. ${ }^{31}$ In this study, we sought to explore the risk factors associated with DR and STDR in mainland China.

\section{METHODS}

\section{Research design}

The Lifeline Express Diabetic Retinopathy Screening Program was conducted nationwide, and it involved a cross-sectional investigation in eight hospitals from six different provinces (Shandong, Henan, Inner Mongolia, Jilin, Guangxi and Guangdong Provinces). Subjects were recruited from hospitals and local communities (1/3 from hospital patients, $1 / 3$ from city residents and the other 1/3 from rural residents) between April 2014 and October 2015. The study protocol was approved by the Peking University Third Hospital Ethics Committee, and written informed consent was obtained for each subject. The study was performed in accordance with the Declaration of Helsinki.

In the hospital, subjects were diagnosed with DM by qualified physicians and transferred to eight screening clinics. In the community, subjects were recruited by advertisement, and medical records of a DM diagnosis were required when they visited the screening clinics. Of all the screening clinics, three were in the south and five were in the north of China. All subjects received a digital, colourful and non-stereoscopic retinography, which was taken by a non-mydriatic auto fundus camera. The photograph included two fields for each eye: one centred at the optic disc and the other centred at the macula.

\section{DR/STDR diagnosis and grading}

DR was graded by trained and certified optometrists and ophthalmologists at the Lifeline Express Diabetic Retinopathy Central Assurance Centre. All of the graders underwent periodic tests to ensure the accuracy of their grading. Retinopathy was graded according to fundus photographs of two eyes into no DR (R0) and DR (other stages), and DR was also graded as none sight-threatening diabetic retinopathy (non-STDR) or sight-threatening diabetic retinopathy (STDR) according to the UK guidelines. ${ }^{32}$ Non-STDR was recognised as R0 and R1, while STDR was identified as present if any features of maculopathy (M1), preproliferative diabetic retinopathy (R2) or proliferative diabetic retinopathy (R3) were found. If the fundus photographs were ungradable because of missing data or non-diagnostic images due to cataracts or vitreous opacities, the patients were excluded from the risk factor analysis. If the photograph of one eye was unrecognised, the final diagnosis was determined by the only remaining photograph. In this condition, if the remaining photograph was graded R0, patients were excluded because of a lack of evidence. If the remaining photograph was graded R1, patients were diagnosed as DR and excluded from the STDR analysis, while if it was graded as M1, R2 or R3, patients were diagnosed as DR and STDR.

\section{Information collection}

At the time of the clinical visit, the gender, age, diagnosis age, diabetes duration (calculated from the age and onset age), diabetes type (as evaluated by physicians in screening clinics), body mass index (BMI; calculated from the measured height and weight), waist-hip ratio (WHR; calculated from the measured waistline and hipline) and type of treatment were recorded. Systolic blood pressure (SBP), diastolic blood pressure (DBP), fasting blood glucose (FBG), postprandial blood glucose (PBG) 2 hours after eating $75 \mathrm{mg}$ glucose and glycosylated haemoglobin (HbA1c) were measured at the screening clinics, and blood samples after fasting for 8 hours were collected for cholesterol, triglyceride, high-density lipoprotein (HDL), low-density lipoprotein (LDL), blood urea nitrogen (BUN) and serum creatinine $(\mathrm{Cr})$ measurements. Gender, age, diagnosis age, diabetes duration, blood pressure (BP), FBG and HbAlc were collected for each patient, while other information was limited to only part of the subjects because of the environment and devices.

\section{Statistical analysis}

Statistical analyses were performed using SPSS V.18.0. The independent t-test was used to compare continuous variables, and the $\chi^{2}$ test was used to compare discontinuous variables among the groups. Owing to the limited number of patients with type 1 and gestational diabetes, we analysed the data from patients with type 2 diabetes only.

We first conducted a four-step analysis of the relationship between the risk factors and DR. In the first step, the mean values and the median values of the main variables were calculated. In the second step, univariate analyses of the associations between the existence of DR and the risk factors were completed. In this step, several continuous variables, including age, diabetes duration, BP, BMI and WHR, were also transferred into categorical variables, to explore their detailed relationship with DR. Age was divided into groups with 10-year intervals, and diabetes 
duration was divided into groups with 5-year intervals. BP values were catalogues as normal $\mathrm{BP}$, level 1 hypertension, level 2 hypertension and severe hypertension. ${ }^{33}$ BMI was divided into underweight $(<18.5)$, normal weight $(\geq 18.5$ and $<24)$, overweight $(\geq 24$ and $<28)$ and obese $(\geq 28)$ categories. WHR was divided into normal WHR (male $\leq 0.90$ and female $\leq 0.85$ ) and abdominal obesity (male 0.90 and female $\leq 0.85$ ) and was also divided into male and female groups. In the third step, multicollinearity diagnosis was performed, and a variance inflation factor $>10$ was thought to have a high collinearity. ${ }^{34}$ Furthermore, variables with high a collinearity were evaluated, and the variable that was most relevant to the research purpose was determined by two researchers (JY and YL). In the fourth step, binary logistic regression analyses were carried out, taking the existence of DR as the dependent variable and all risk factors, which were significantly associated with the existence of DR in the former step or considered to be important risk factors based on existing studies, as independent variables. Owing to limitations in the information collection, we separately analysed the eight risk factors that were completely collected in each screening clinic (eight-factor analysis) and all risk factors (all-factor analysis); furthermore, the differences between the two analyses were discussed.

Then, the relationship between the risk factors and STDR was also conducted in the aforementioned way. ORs and $95 \%$ CIs were calculated. An $\alpha$ level of 0.05 was adopted as the significance level.

At last, nomograms for DR and STDR risk factors were developed, and significant risk factors in former binary logistic regression were regarded as predictors. Interpretation of the nomogram in the prediction of DR has been reported in former studies, ${ }^{35}$ which included two major parts. In the first part, the exact values of each predictor were vertically linked to a certain point (the first row of the nomogram), and the total points of each predictor were calculated. In the second part, the total points were linked to a specific risk incidence of DR and STDR (the last row of the nomogram), which has implications for clinical practice.

\section{Results}

From April 2014 to October 2015, 13473 patients with DM from six provinces were enrolled in the study. A percentage of 45.9 patients were from the southern provinces $(6180 / 13473)$ and $54.1 \%$ were from the northern provinces $(7293 / 13473)$. Of all the patients, 13304 patients were diagnosed with type 2 diabetes, 96 were diagnosed with type 1 diabetes and 73 were diagnosed with gestational diabetes. Patients were divided into a no DR and a DR group and a non-STDR and a STDR group, according to the fundus photograph grading. Five hundred and seventy-one patients were excluded from the DR risk factor analysis, and 683 patients were excluded from the STDR risk factor analysis because of the diagnostic rules mentioned above. Finally, 12733 patients were included in the DR risk factor analysis, and
12621 patients were included in the STDR risk factor analysis (shown in figure 1).

First, analyses of the DR risk factors were performed, and the basic characteristics of all risk factors are shown in table 1 . The results of the univariate analyses indicated that the age, diagnosis age, diabetes duration, SBP, DBP, waistline, hipline, WHR, medicine type (oral medication or insulin injection), FBG, PBG, HbAlc, BUN, and LDL were statistically significantly different between the groups $(\mathrm{p}<0.05)$, and no significant difference was found in the gender, BMI, Cr, cholesterol, triglyceride and HDL $(\mathrm{p}>0.05)$.

Furthermore, our categorical analyses showed that patients were getting less likely to suffer from DR every 10 years after 60 years of age, while no difference was found before age 60 . The incidence of DR increased significantly for every 5 years of diabetes duration but stopped increasing after 20 years of diabetes duration. The results of the BP analysis indicated that diabetes incidence increased with increases in the BP, although diabetes incidence did not differ between level $3 \mathrm{BP}$ and level $2 \mathrm{BP}$. Females had a higher WHR in DR, while males did not, and the condition of abdominal obesity did not influence the incidence of DR.

Then, multivariate analyses were performed. Multicollinearity diagnosis was performed in both the eight-factor analysis and all-factor analysis. The results excluded the diagnosis age (highly correlated to the age and DR duration) in the eight-factor analysis and excluded the diagnosis age (highly correlated to age and DR duration), waistline and hipline (both of which were highly correlated to WHR) in the all-factor analysis because of the high collinearity. Multiple logistic regression analyses were carried out, and the results are shown with the STDR analysis. The results of eight-factor analysis (with the diagnosis age excluded) showed that younger age, longer diabetes duration, higher SBP, higher FBG and higher HbAlc were independent risk factors for DR $(p<0.05)$, and sex and DBP were not significantly associated with DR ( $>0.05)$. A multiple logistic regression of the all-factor analysis (with diagnosis age, waistline, and hipline excluded) was also conducted, and the results showed that younger age, longer diabetes duration, higher SBP, HbAlc, PBG, oral medicine, insulin use, higher triglyceride and higher LDL were the independent risk factors for DR $(p<0.05)$, while sex, DBP, BMI, FBG, WHR, BUN, Cr, cholesterol and HDL were not associated with DR $(\mathrm{p}>0.05)$.

After the DR risk factors analyses, analyses of the STDR risk factors were conducted, and characteristics of the risk factors are shown in table 2. Age, diagnosis age, diabetes duration, SBP, DBP, HbA1c, FBG, PBG, waistline, WHR, medicine, $\mathrm{Cr}$ and LDL showed statistically significant differences between groups $(\mathrm{p}<0.05)$, while gender, BMI, hipline, BUN, cholesterol, triglyceride and HDL were not significantly different. After a multicollinearity diagnosis, the diagnosis age was excluded in the eight-factor analysis. The diagnosis age, waistline and hipline were 




Figure 1 Flow diagram of the data processing. DR, diabetic retinopathy; STDR, sight-threatening diabetic retinopathy.

excluded in the all-factor analysis. The results of multiple logistic regressions of STDR analyses (together with DR analyses) are shown in table 3 . The results of the eightfactor analysis (with the diagnosis age dropped) showed that younger age, longer diabetes duration, higher SBP, DBP, FBG and HbAlc were independent risk factors for STDR $(p<0.05)$, and sex was not significantly associated with STDR ( $p>0.05)$. The results of the all-factor analysis indicated that a younger age, longer diabetes duration, higher SBP, higher FBG, oral medicine use and insulin use were regarded as independent risk factors for STDR $(p<0.05)$, while other risk factors showed no significant differences $(\mathrm{p}>0.05)$.

Furthermore, we subcategorised the non-STDR group into the non-DR and DR but not STDR groups, and the risk factors between the DR but not STDR group and the non-DR, STDR or DR group. The results showed that independent risk factors for DR but not STDR compared with non-DR were exactly the same as for DR/no DR. However, the risk factors of the STDR compared with DR but not STDR analysis showed two new independent risk factors in addition to those for STDR/non-STDR, which were male sex and Cr.

Finally, we developed a nomogram to simplify the presentation and understanding of our results (figure 2).

\section{DISCUSSION}

Based on the results of our study, we tried to find a reasonable explanation and an internal relationship between DR, STDR and risk factors.

First, focusing on the univariate analysis, 14 out of 20 risk factors were found to be significantly different between the non-DR and DR groups, and 13 out of 20 factors were found to be different between the non-STDR and STDR groups. Basically, all risk factors were divided into non-modifiable and modifiable risk factors. Non-modifiable factors included gender, age, diagnosis age and diabetes duration. In both the non-DR/DRand the non-STDR/STDR analyses, gender showed no significant differences. This was also reported in several previous studies ${ }^{10}{ }^{12}$ while the results of some studies remained controversial. ${ }^{11}{ }^{13}$ Significantly younger age was observed in the DR and STDR groups, and a longer duration of diabetes was also found in the DR and STDR groups. Longer duration may represent a longer period of retinal toxicity induced by high glucose levels, which is believed to be associated with both vascular and neural death in the retina. ${ }^{36}$ The existing studies show an older or much younger age in the DR patients than in the non-DR patients. ${ }^{26}$ In our study, an older age seemed to be a protective variable for DR but was instead found to be a 
Table 1 Univariate analysis of DR risk factors $(n=12733)$

\begin{tabular}{|c|c|c|c|c|c|c|c|}
\hline Variable & $\mathbf{n}$ & $\begin{array}{l}\text { No DR } \\
(\mathrm{n}=8772)\end{array}$ & $\mathbf{n}$ & $\begin{array}{l}\text { DR } \\
(n=3961)\end{array}$ & $\mathbf{p}$ & OR & $95 \% \mathrm{Cl}$ \\
\hline Male gender, n (\%) & 8772 & $3985(45.4)$ & 3961 & $1851(46.7)$ & 0.172 & & \\
\hline Age, years (SD) & 8770 & $63.0(10.3)$ & 3959 & $61.0(9.8)$ & $<0.001$ & & \\
\hline$<30, \%$ & 11 & 30.6 & 25 & 69.4 & & & \\
\hline $30-40, \%(v s<30)$ & 143 & 66.8 & 71 & 33.2 & 0.757 & 1.128 & 0.526 to 2.423 \\
\hline 40-50, \% (vs 30-40) & 733 & 64.2 & 408 & 35.8 & 0.469 & 1.121 & 0.823 to 1.527 \\
\hline 50-60, \% (vs 40-50) & 2138 & 63.2 & 1243 & 36.8 & 0.542 & 1.004 & 0.908 to 1.201 \\
\hline 60-70, \% (vs 50-60) & 3443 & 69.7 & 1494 & 30.3 & $<0.001$ & 0.746 & 0.680 to 0.819 \\
\hline$>70, \%$ (vs 60-70) & 2217 & 76.0 & 700 & 24.0 & $<0.001$ & 0.728 & 0.655 to 0.808 \\
\hline Diagnosis age, years (SD) & 8770 & $56.3(10.2)$ & 3959 & $50.8(10.3)$ & $<0.001$ & & \\
\hline Diabetes duration, years (SD) & 8772 & $6.7(5.9)$ & 3961 & $10.2(6.8)$ & $<0.001$ & & \\
\hline$<5, \%$ & 4692 & 80.0 & 1174 & 20.0 & & & \\
\hline $5-10, \%(v s<5)$ & 2314 & 65.7 & 1208 & 34.3 & $<0.001$ & 2.086 & 1.898 to 2.293 \\
\hline $10-15, \%$ (vs 5-10) & 1032 & 57.6 & 761 & 42.4 & $<0.001$ & 1.413 & 1.257 to 1.587 \\
\hline $15-20, \%$ (vs 10-15) & 521 & 48.2 & 560 & 51.8 & $<0.001$ & 1.458 & 1.252 to 1.696 \\
\hline$>20, \%$ (vs 15-20) & 213 & 45.2 & 258 & 54.8 & 0.281 & 1.127 & 0.907 to 1.400 \\
\hline BMI (SD) & 6000 & $24.7(3.5)$ & 2854 & $24.9(3.9)$ & 0.116 & & \\
\hline Underweight, \% & 97 & 64.7 & 53 & 35.3 & & & \\
\hline $\begin{array}{l}\text { Normal weight, \% } \\
\text { (vs underweight) }\end{array}$ & 3348 & 68.5 & 1543 & 31.5 & 0.326 & 0.843 & 0.600 to 1.185 \\
\hline $\begin{array}{l}\text { Overweight, \% } \\
\text { (vs normal weight) }\end{array}$ & 2175 & 67.6 & 1043 & 32.4 & 0.414 & 1.041 & 0.946 to 1.145 \\
\hline $\begin{array}{l}\text { Obese, \% } \\
\text { (vs overweight) }\end{array}$ & 380 & 63.9 & 215 & 36.1 & 0.076 & 1.180 & 0.983 to 1.417 \\
\hline SBP, mm Hg (SD) & 8762 & $133.3(16.5)$ & 3952 & $137.0(17.9)$ & $<0.001$ & & \\
\hline DBP, mm Hg (SD) & 8762 & $79.6(9.9)$ & 3952 & $80.8(10.8)$ & $<0.001$ & & \\
\hline Normal BP, \% & 5084 & 72.3 & 1950 & 27.7 & & & \\
\hline $\begin{array}{l}\text { BP level 1, \% } \\
\text { (vs normal) }\end{array}$ & 2840 & 66.7 & 1420 & 33.3 & $<0.001$ & 1.303 & 1.200 to 1.414 \\
\hline $\begin{array}{l}\text { BP level 2, \% } \\
\text { (vs level 1) }\end{array}$ & 665 & 59.3 & 456 & 40.7 & $<0.001$ & 1.371 & 1.198 to 1.569 \\
\hline $\begin{array}{l}\text { BP level 3, \% } \\
\text { (vs level 2) }\end{array}$ & 173 & 57.9 & 126 & 42.1 & 0.648 & 1.062 & 0.820 to 1.376 \\
\hline Waistline, cm (SD) & 5719 & $89.3(10.1)$ & 2735 & $90.3(10.6)$ & $<0.001$ & & \\
\hline Hipline, cm (SD) & 5719 & $96.6(9.7)$ & 2735 & $97.1(9.5)$ & 0.028 & & \\
\hline WHR (SD) & 5719 & $0.926(0.074)$ & 2735 & $0.930(0.069)$ & 0.007 & & \\
\hline Abdominal obesity, n (\%) & & 4677 (81.8) & & 2267 (82.9) & 0.213 & 1.079 & 0.957 to 1.217 \\
\hline Female (SD) & 3152 & $0.915(0.076)$ & 1501 & $0.923(0.072)$ & 0.002 & & \\
\hline Male (SD) & 2567 & $0.94(0.070)$ & 1234 & $0.94(0.064)$ & 0.674 & & \\
\hline Medicine & 5793 & & 2797 & & $<0.001$ & & \\
\hline No medicine, \% & 660 & 87.0 & 99 & 13.0 & & & \\
\hline $\begin{array}{l}\text { Oral medicine, } \% \\
\text { (vs no medicine) }\end{array}$ & 3296 & 73.2 & 1208 & 26.8 & $<0.001$ & 5.407 & 4.331 to 6.752 \\
\hline $\begin{array}{l}\text { Insulin, \% } \\
\text { (vs oral medicine) }\end{array}$ & 1837 & 55.2 & 1490 & 44.8 & $<0.001$ & 2.213 & 2.013 to 2.434 \\
\hline $\mathrm{FBG}, \mathrm{mmol} / \mathrm{L}(\mathrm{SD})$ & 7547 & $7.8(2.4)$ & 3517 & $8.7(3.0)$ & $<0.001$ & & \\
\hline PBG, mmol/L (SD) & 4780 & $10.7(3.3)$ & 2095 & $11.8(3.5)$ & $<0.001$ & & \\
\hline
\end{tabular}


Table 1 Continued

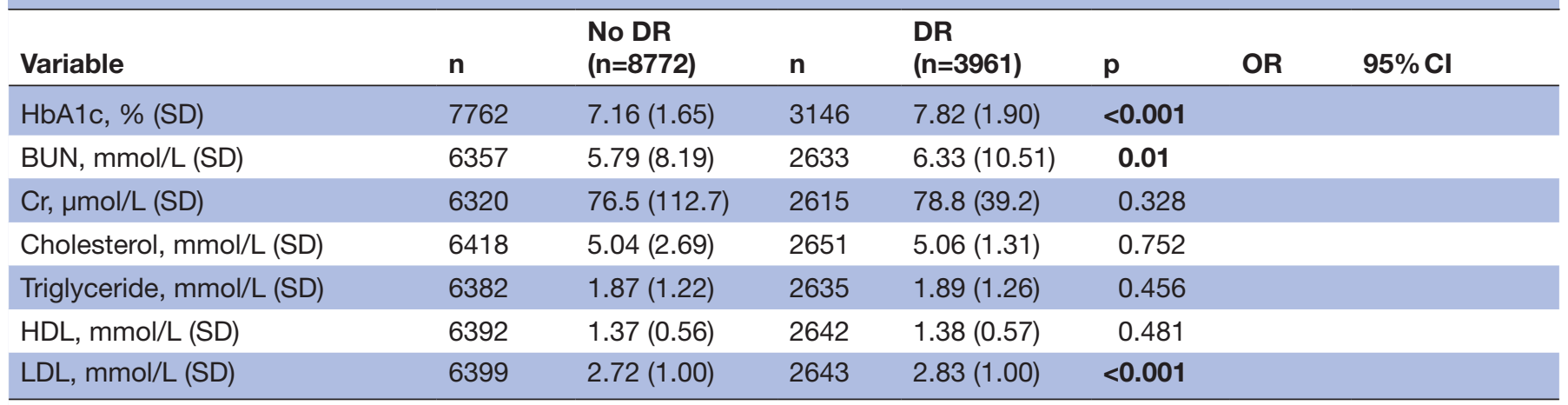

Continuous variables were reported as mean value and SD, and categorical variables were reported as percentage, $\mathrm{OR}$ and $95 \% \mathrm{Cl}$. $\mathrm{p}<0.05$ was considered statistically significant and marked in bold.

$\mathrm{BMI}$, body mass index; BP, blood glucose; BUN, blood urea nitrogen; $\mathrm{Cr}$, creatinine; DBP, diastolic blood pressure; DR, diabetic retinopathy; FBG, fasting blood glucose; HbA1c, glycosylated haemoglobin; HDL, high-density lipoprotein; LDL, low-density lipoprotein; n, number; PBG, postprandial blood glucose; SBP, systolic blood pressure; WHR, waist-hip ratio.

variable for STDR, especially in patients with DM older than 60 . This means that even though older age was associated with a lower incidence of DR, it is associated with a greater threat to vision. We thought that this phenomenon might be explained by the higher mortality risk in older DR populations. However, as DR was correlated with severe general diseases ${ }^{37}$ this result might be related to survival bias. However, older age also implied a longer suffering of hyperglycaemia, which might be more vision threatening. Furthermore, we explored the relationship between age and $\mathrm{HbAlc}$, diabetes duration and the therapeutic regimen. The results indicated that with increasing age, the diabetes duration increased, while the HbA1c and use of insulin decreased. This implied that although the duration of diabetes increased, older people had a better glucose management and required milder medicine. In this way, age was determined to be a protective factor.

Modifiable risk factors included the obesity index, BP, medicine, blood glucose, renal function and blood lipid levels. Both DR and STDR showed a significantly higher WHR, BP, blood glucose level, LDL and a higher incidence of insulin use than the non-DR and non-STDR groups. BUN was only significantly higher in the DR group than the non-DR group, meanwhile Cr was only significantly higher in the STDR than in the non-STDR group. WHR was thought to be associated with $\mathrm{DM}^{38}$ and was also thought to be a risk factor for severe DR in women. ${ }^{39}$ Our study showed similar results in the univariate analysis. High BP indicated a significantly higher incidence of DR and STDR, while the effects did not increase after a certain level. Blood glucose and LDL were significantly higher in the DR and STDR groups than in the non-DR and non-STDR groups, while the cholesterol level showed no significant difference, indicating that the DR and STDR groups had poor management of blood glucose and LDL. BUN and $\mathrm{Cr}$ were both common variables that reflected renal function, and our study indicated that DR and STDR showed a higher level of renal injury than the non-DR and non-STDR groups. The application of oral medicine or insulin was also reported to be a risk factor in the former studies, perhaps because of the severity of the disease condition. ${ }^{11}$

Second, the results of multiple logistic regression analyses showed that independent risk factors of DR and STDR were similar in the eight-factor analysis (with the diagnosis age excluded) and were different in the all-factor analysis (with diagnosis age, waistline and hipline excluded). In the all-factor analysis, younger age, diabetes duration and SBP were found to be independent risk factors for both DR and STDR, while PBG, HbAlc, triglyceride and LDL were found to be independent risk factors for DR only, and FBG was found to be an independent risk factor for STDR only. Age, diabetes duration and SBP were reported to be independent risk factors for DR or DR progression, ${ }^{10} 2025$ while differences in the blood glucose were harder to explain. HbAlc has been reported to be an independent risk factor in the development and progression of DR in earlier studies, ${ }^{18}{ }^{21}$ but there is little evidence on the role of PBG in DR progression. HbA1c has long been considered to represent the management condition of blood glucose, and bad glucose management is known to contribute to the occurrence and progression of DR. ${ }^{9-11}$ PBG was reported to be abnormal in $31 \%$ of patients with DM whose FBG was normal, ${ }^{40}$ so it was considered to be an important diagnostic factor for DM. PBG was shown to be more valuable in the prediction of ischaemic and haemorrhagic stroke and cardiovascular disease mortality, while FBG showed only weak predictive power. ${ }^{414}$ Therefore, we thought that it was reasonable that PBG was a risk factor for severe complications of DM, such as DR. One possible mechanism of PBG in the progression of DR might be that PBG reflects the capacity of insulin secretion, the peak of which was shown to be delayed in type $2 \mathrm{DM}^{43}$. High levels of PBG indicate that insulin secretion is relatively insufficient, which might result in a blood glucose fluctuation after food intake and subsequent harm to the targeted organs. Our study first found that FBG was an independent risk factor for STDR, although we note that the OR was only 1.043 for 


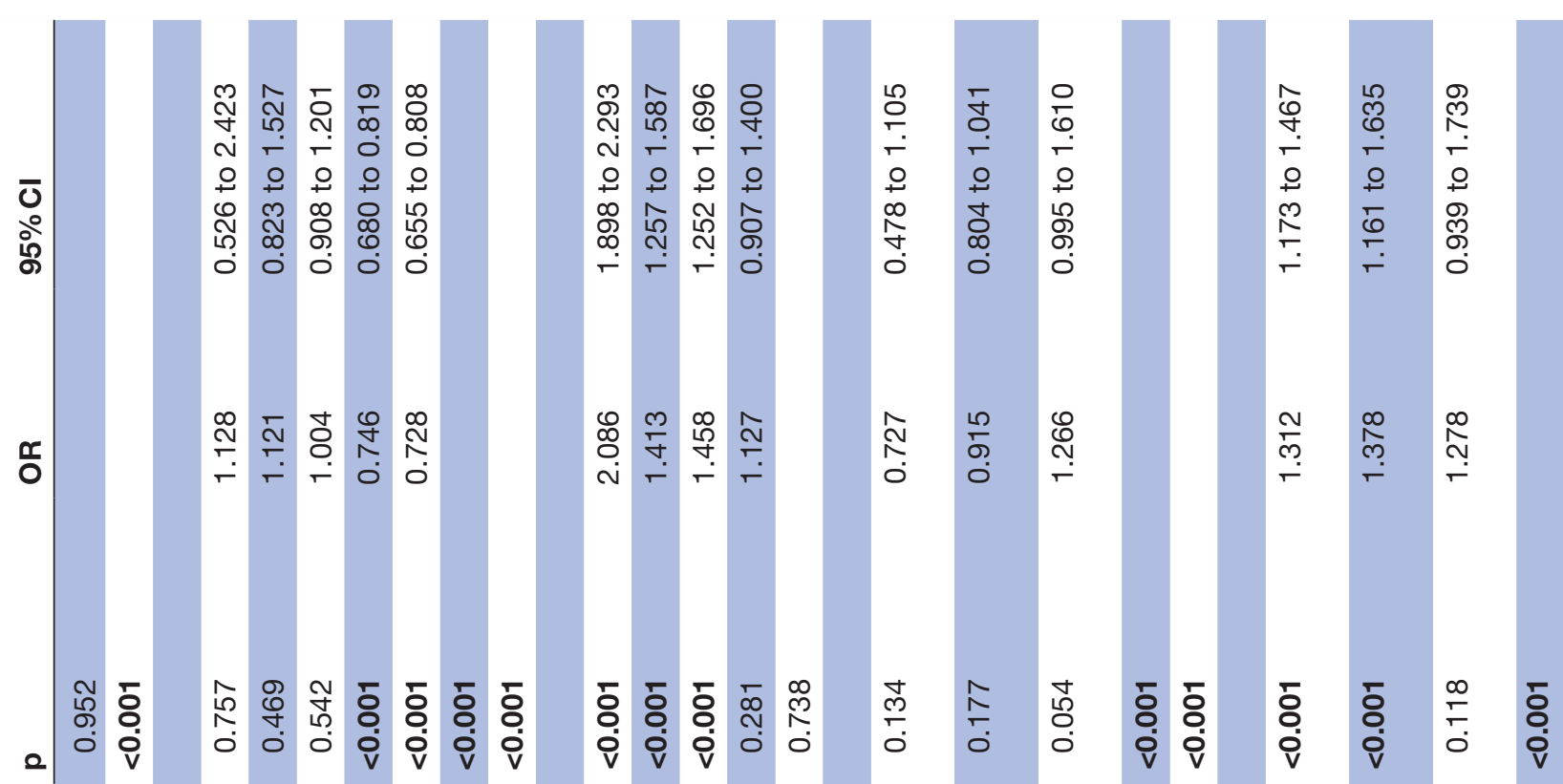

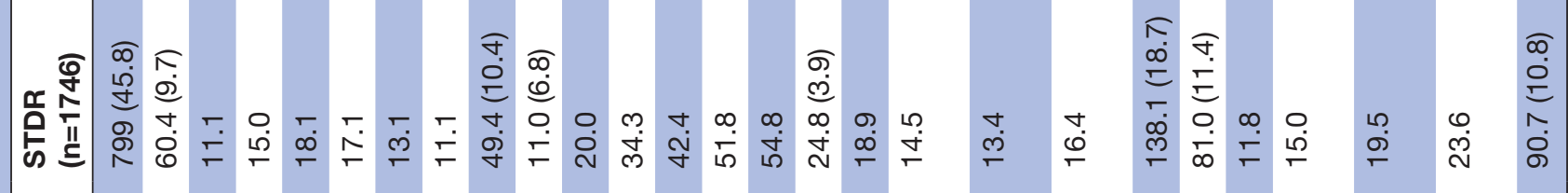

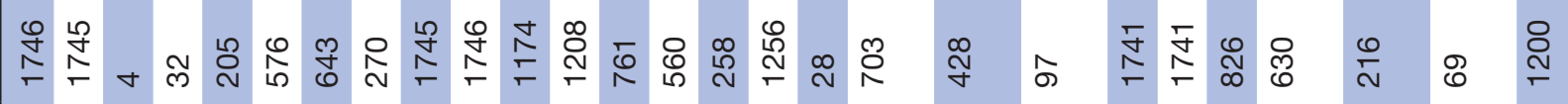

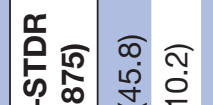

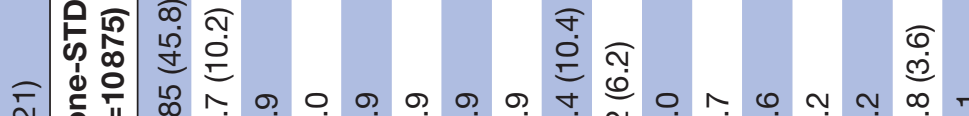

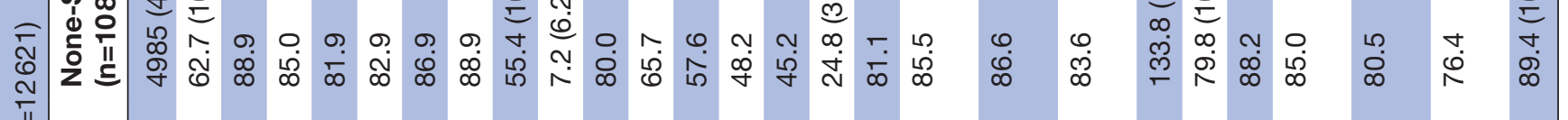



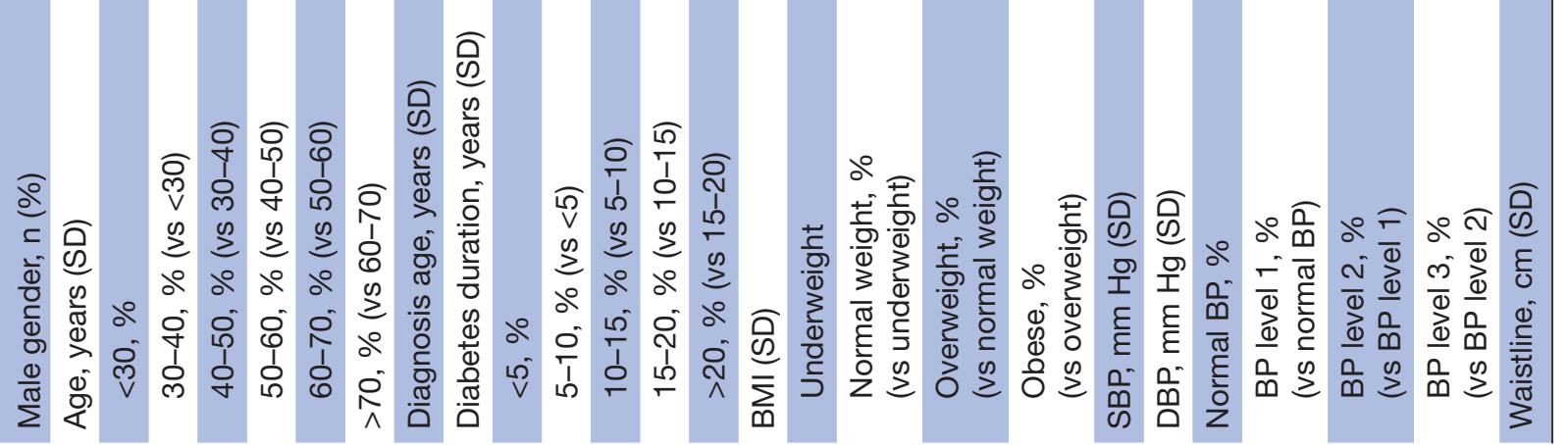

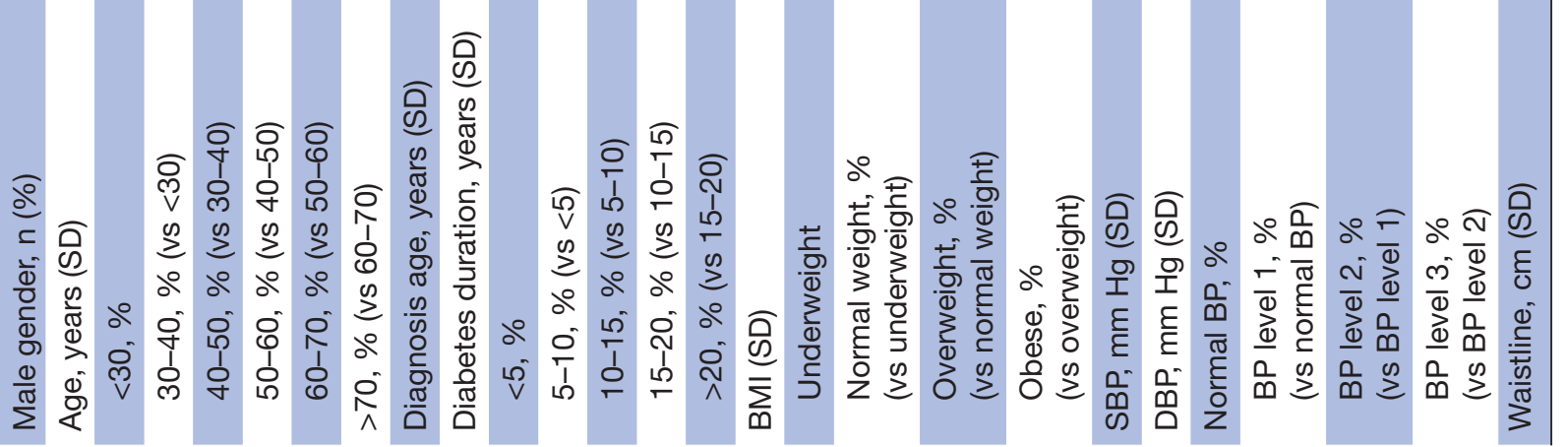

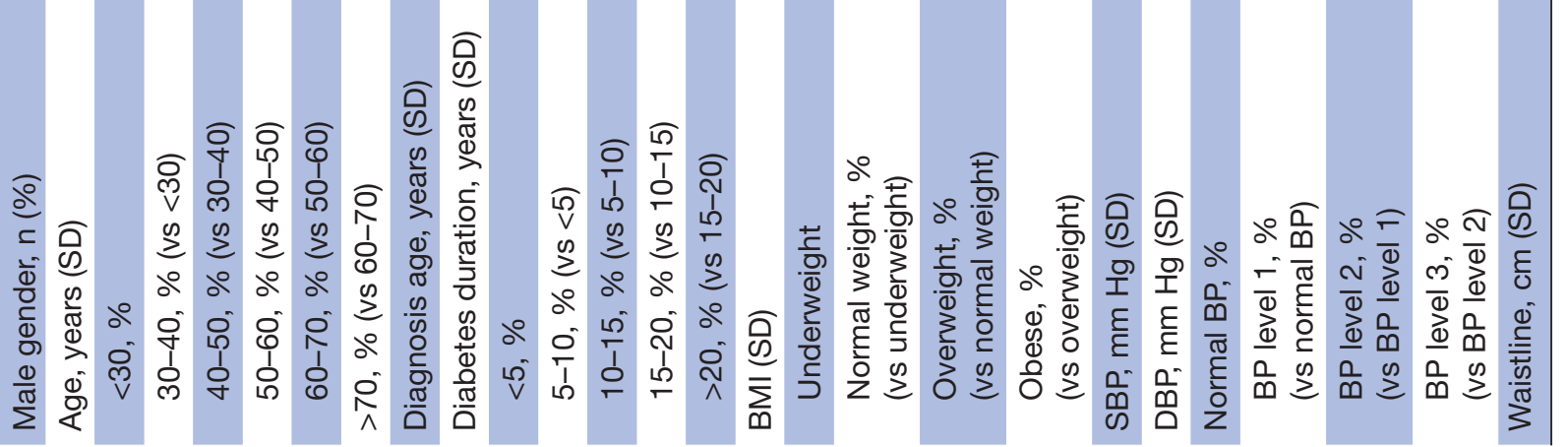

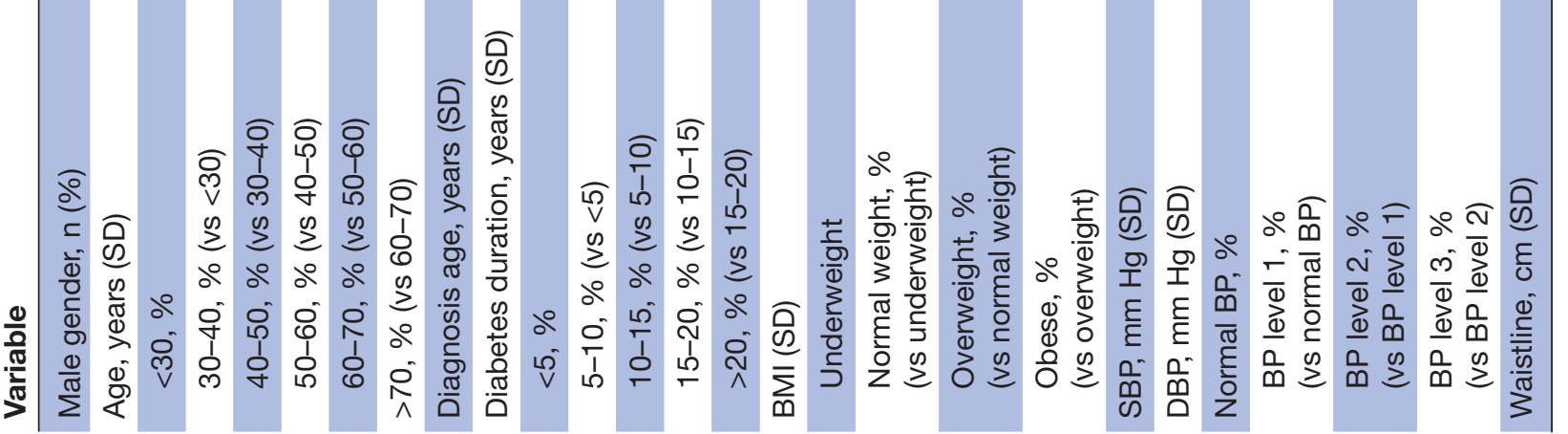

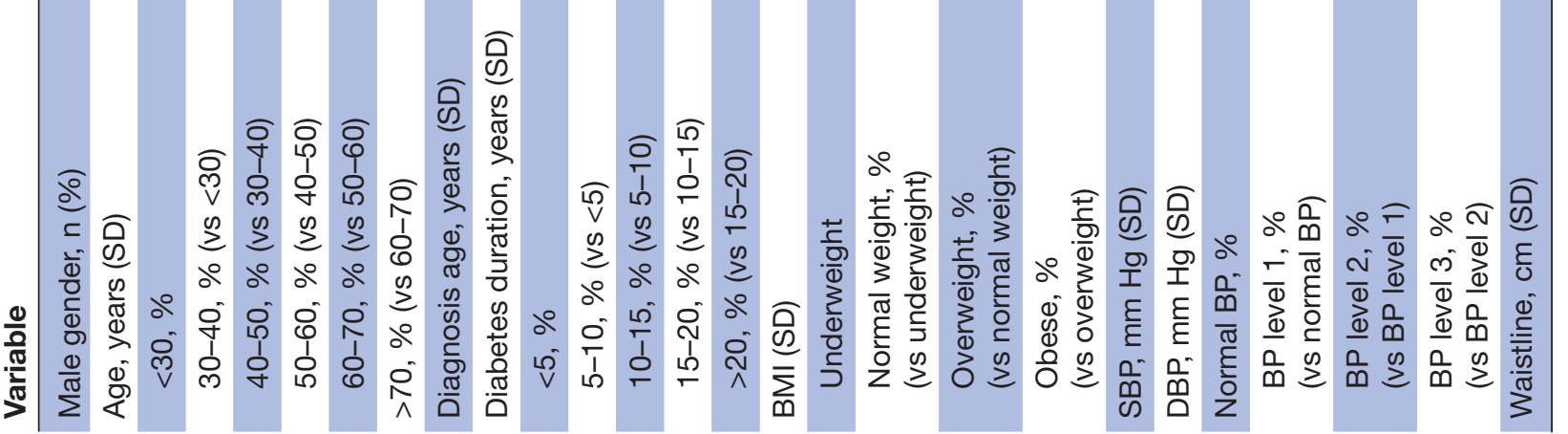




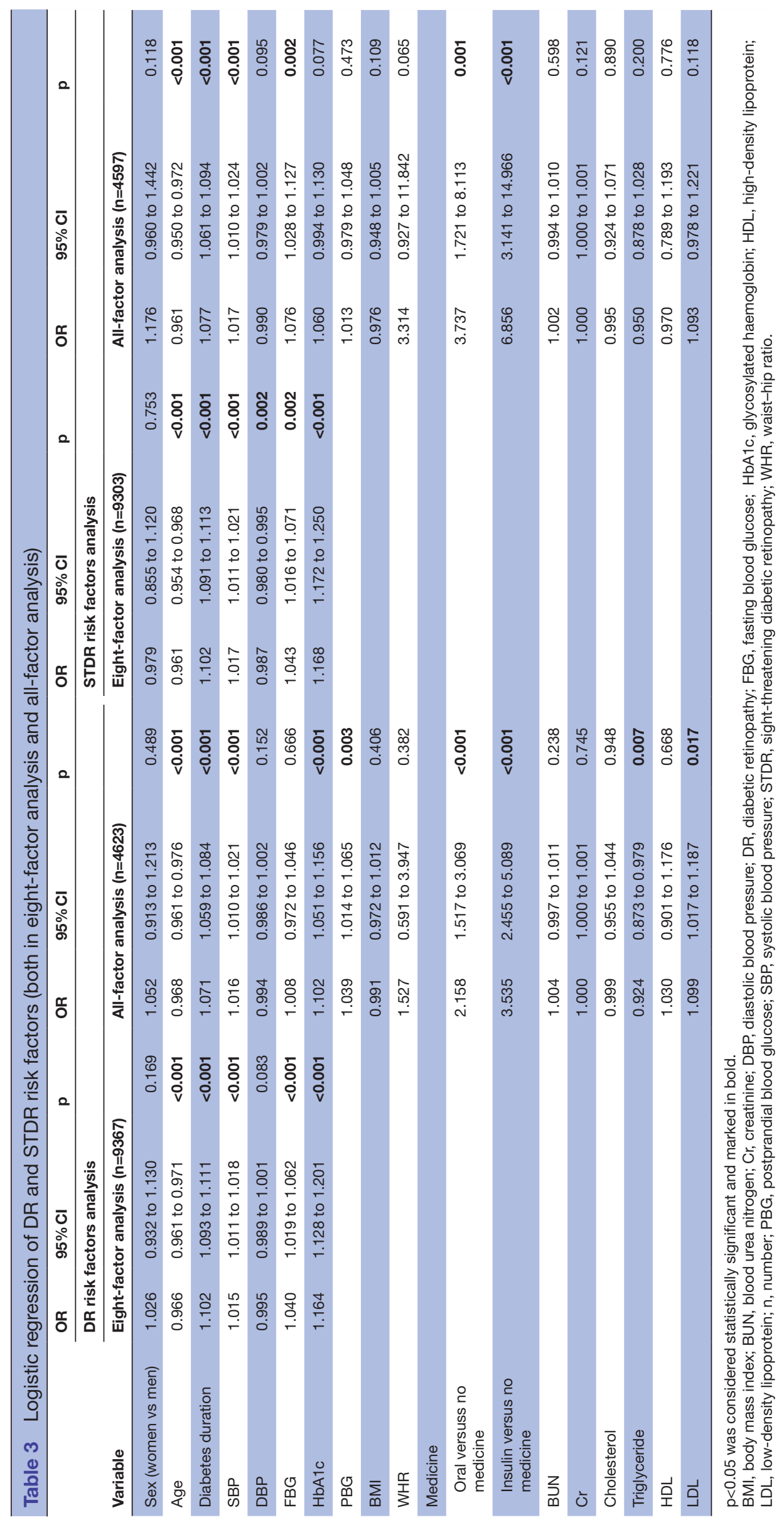


A

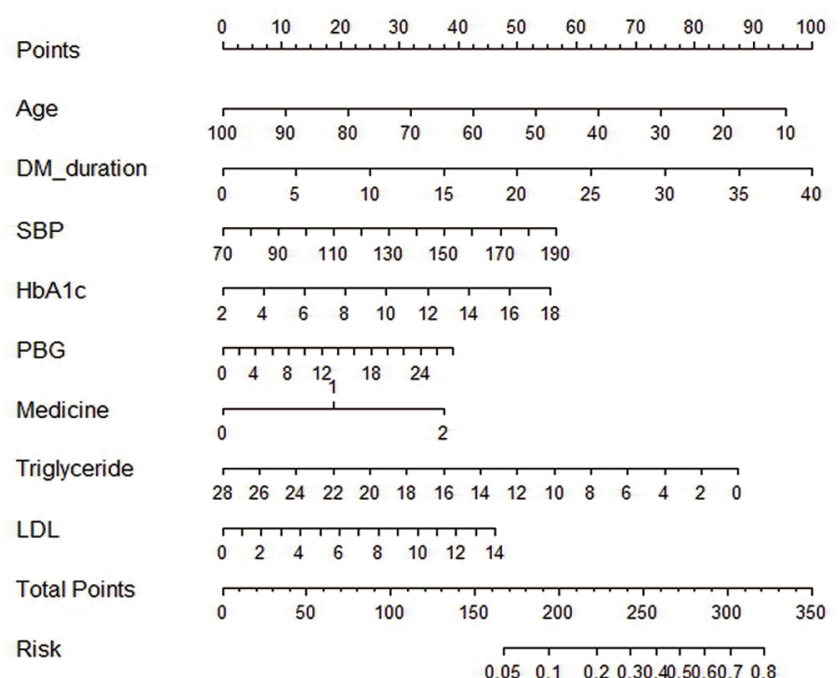

B

Points
Age
DM_duration
SBP
FBG
Medicine
Total Points
Risk


\begin{tabular}{lllllllllllllll}
\hline 2 & 4 & 6 & 8 & 10 & 12 & 14 & 16 & 18 & 20 & 22 & 24
\end{tabular}


$\begin{array}{lllllll}0.01 & 0.05 & 0.1 & 0.2 & 0.3 & 0.4 & 0.50 .60 .7\end{array}$

Figure 2 Nomograms for DR (A) and STDR (B) risk factors. Risk factors were chosen based on results of logistic regression analysis. Each risk factor of the patient was assessed on basis of the nomogram and got a point by vertically corresponding to the first line. Aggregated points of each risk factor corresponded to a particular occurrence probability of DR or STDR in the last line. DR, diabetic retinopathy; DM, diabetes mellitus; FBG, fasting blood -glucose; HbA1c, glycosylated haemoglobin; LDL, low-density lipoprotein; PBG, postprandial blood glucose; SBP, systolic blood pressure; STDR, sight-threatening diabetic retinopathy.

a 1 unit increase in FBG and thus has a limited power to predict the incidence of STDR. No existing studies previously showed that FBG was an independent risk factor for DR, which might be caused by a higher predictive value of HbA1c in these studies. Determining a possible mechanism of FBG for STDR required further studies. LDL and triglyceride levels were also independent risk factors for DR, which indicated that the management of blood lipids is very important for DR prevention but was of limited relevance for STDR compared with the other risk factors.

This study explored the risk factors of DR and STDR, which provided some insights for the DR progression in Chinese population, and our findings could be applied nationwide for further DR management. Our study also had some limitations. First, owing to the limitations of the device and screening environment, several variables were incomplete. If we meant to analyse all variables in this study, considerable data would be abandoned, and the results of multivariate regression might be influenced. Second, although we tried to balance patients from north and south and from urban and rural areas, the patients enrolled in the final all-factor logistic regression were not fully balanced because of the exclusion of missing data. Third, patients and screening clinics were not collected via stratified sampling and therefore were not fully representative of patients in mainland China. Fourth, our study design was cross-sectional. Compared with cohort studies, cross-sectional studies provide weaker evidence, and the results must be carefully explained. Fifth, the risk factors explored in this study had been reported in previous studies, and further studies should thus include more risk factors. More cohort studies focusing on several areas of China will therefore be required to expand on these results.

\section{CONCLUSIONS}

Our study demonstrated that age, diabetes duration and SBP were independent risk factors for both DR and STDR. PBG, HbAlc, triglyceride and LDL were independent risk factors for DR only, and FBG was an independent risk factor for STDR only. These results are similar to the results of existing studies and may provide some evidence for the clinical prevention of DR and STDR, especially as FBG, PBG, and HbAlc were all important predictors for the occurrence or progression of DR, requiring stricter glucose control. More DR screening and information collection are required, which may decrease the incidence of DR and improve clinical results.

Collaborators Chinese Foundation for Lifeline Express and Lifeline Express Hong Kong Foundation.

Contributors YL and JY designed the whole study, completed the data collection and wrote the manuscript. JY and LT performed the data analysis, and LT contributed to the epidemic portion of the manuscript. $\mathrm{HL}$ and $\mathrm{XJ}$ contributed to the study design and contributed to the writing of the manuscript. MZ assisted with the study design, data collection and contributed to the editing of the manuscript. XL oversaw the study, gave advice on the study design and revised the manuscript. XL was the guarantor of this study and had full access to all the data in this study. XL also takes responsibility for the integrity of the data and the accuracy of the data analysis.

Funding This study was also supported by the Beijing Municipal Science \& Technology Commission. Grant Number: Z141107002514042.

Competing interests None declared.

Patient consent Obtained. 
Ethics approval Peking University Third Hospital Ethics Committee.

Provenance and peer review Not commissioned; externally peer reviewed.

Data sharing statement № additional data are available.

Open Access This is an Open Access article distributed in accordance with the Creative Commons Attribution Non Commercial (CC BY-NC 4.0) license, which permits others to distribute, remix, adapt, build upon this work non-commercially, and license their derivative works on different terms, provided the original work is properly cited and the use is non-commercial. See: http://creativecommons.org/ licenses/by-nc/4.0/

(c) Article author(s) (or their employer(s) unless otherwise stated in the text of the article) 2017. All rights reserved. No commercial use is permitted unless otherwise expressly granted.

\section{REFERENCE}

1. King H, Aubert RE, Herman WH. Global burden of diabetes, 19952025: prevalence, numerical estimates, and projections. Diabetes Care 1998;21:1414-31.

2. Bourne RR, Stevens GA, White RA, et al. Causes of vision loss worldwide, 1990-2010: a systematic analysis. Lancet Glob Health 2013;1:e339-49.

3. Jones S, Edwards RT. Diabetic retinopathy screening: a systematic review of the economic evidence. Diabet Med 2010;27:249-56.

4. Heintz E, Wiréhn AB, Peebo BB, et al. Prevalence and healthcare costs of diabetic retinopathy: a population-based register study in Sweden. Diabetologia 2010;53:2147-54

5. Moriya T, Tanaka S, Sone H, et al. Patients with type 2 diabetes having higher glomerular filtration rate showed rapid renal function decline followed by impaired glomerular filtration rate: Japan Diabetes Complications Study. J Diabetes Complications 2017;31.

6. de Moraes G, Layton CJ. Therapeutic targeting of diabetic retinal neuropathy as a strategy in preventing diabetic retinopathy. Clin Exp Ophthalmol 2016;44:838-52.

7. Lim Y, Chun S, Lee JH, et al. Association of bone mineral density and diabetic retinopathy in diabetic subjects: the 2008-2011 Korea National Health and Nutrition Examination Survey. Osteoporos Int 2016;27:2249-57.

8. Kawasaki R, Tanaka S, Abe S, et al. Risk of cardiovascular diseases is increased even with mild diabetic retinopathy: the Japan Diabetes Complications Study. Ophthalmology 2013;120:574-82.

9. Knudsen LL, Lervang HH, Lundbye-Christensen $\mathrm{S}$, et al. The North Jutland County Diabetic Retinopathy Study: population characteristics. Br J Ophthalmol 2006;90:1404-9.

10. Bertelsen $\mathrm{G}$, Peto $\mathrm{T}$, Lindekleiv $\mathrm{H}$, et al. Tromsø eye study: prevalence and risk factors of diabetic retinopathy. Acta Ophthalmol 2013;91:716-21.

11. Zhang X, Saaddine JB, Chou CF, et al. Prevalence of diabetic retinopathy in the United States, 2005-2008. JAMA 2010;304:649-56.

12. Piermarocchi R, Piermarocchi S, Tognetto D, et al. Prevalence of diabetic retinopathy and its risk factors in the PAMDI population of the mediterranean basin. Eur J Ophthalmol 2015;25:e22.

13. George M, Harper R, Balamurugan A, et al. Diabetic retinopathy and its risk factors in a population-based study. J Prim Care Community Health 2011;2:122-6.

14. Pedro RA, Ramon SA, Marc BB, et al. Prevalence and relationship between diabetic retinopathy and nephropathy, and its risk factors in the North-East of Spain, a population-based study. Ophthalmic Epidemiol 2010;17:251-65.

15. Dedov I, Maslova O, Suntsov Y, et al. Prevalence of diabetic retinopathy and cataract in adult patients with type 1 and type 2 diabetes in Russia. Rev Diabet Stud 2009;6:124-9.

16. Pugliese G, Solini A, Zoppini G, et al. High prevalence of advanced retinopathy in patients with type 2 diabetes from the Renal Insufficiency And Cardiovascular Events (RIACE) Italian Multicenter Study. Diabetes Res Clin Pract 2012;98:329-37.

17. Villena JE, Yoshiyama CA, Sánchez JE, et al. Prevalence of diabetic retinopathy in Peruvian patients with type 2 diabetes: results of a hospital-based retinal telescreening program. Rev Panam Salud Publica 2011;30:408-14.

18. Esteves JF, Kramer CK, Azevedo MJ, et al. Prevalence of diabetic retinopathy in patients with type 1 diabetes mellitus. Rev Assoc Med Bras 2009;55:268-73.

19. Romero-Aroca P, Baget-Bernaldiz M, Fernandez-Ballart J, et al Ten-year incidence of diabetic retinopathy and macular edema. Risk factors in a sample of people with type 1 diabetes. Diabetes Res Clin Pract 2011;94:126-32.

20. Kajiwara A, Miyagawa H, Saruwatari J, et al. Gender differences in the incidence and progression of diabetic retinopathy among Japanese patients with type 2 diabetes mellitus: a clinicbased retrospective longitudinal study. Diabetes Res Clin Pract 2014;103:e7-10.

21. Tam VH, Lam EP, Chu BC, et al. Incidence and progression of diabetic retinopathy in Hong Kong Chinese with type 2 diabetes mellitus. J Diabetes Complications 2009;23:185-93.

22. Henricsson M, Nyström L, Blohmé G, et al. The incidence of retinopathy 10 years after diagnosis in young adult people with diabetes: results from the nationwide population-based Diabetes Incidence Study in Sweden (DISS). Diabetes Care 2003;26:349-54.

23. Stratton IM, Kohner EM, Aldington SJ, et al. UKPDS 50: risk factors for incidence and progression of retinopathy in Type II diabetes over 6 years from diagnosis. Diabetologia 2001;44:156-63.

24. Á SM, Burgoslunar CD, Arrietablanco FJ, et al. Four-year incidence of diabetic retinopathy in a Spanish Cohort: the MADIABETES study. PLoS One 2012;8:377-80.

25. $\mathrm{Xu} \mathrm{J}, \mathrm{Xu} \mathrm{L}$, Wang $\mathrm{YX}$, et al. Ten-year cumulative incidence of diabetic retinopathy. The Beijing Eye Study 2001/2011. PLoS One 2014:9:e111320.

26. Jones CD, Greenwood RH, Misra A, et al. Incidence and progression of diabetic retinopathy during 17 years of a population-based screening program in England. Diabetes Care 2012;35:592-6.

27. Fundus photographic risk factors for progression of diabetic retinopathy. ETDRS report number 12. Early Treatment Diabetic Retinopathy Study Research Group. Ophthalmology 1991;98(Suppl 5):823-33.

28. Yan ZP, Ma JX. Risk factors for diabetic retinopathy in northern Chinese patients with type 2 diabetes mellitus. Int J Ophthalmol 2016;9:1194-9.

29. Lee R, Wong TY, Sabanayagam C. Epidemiology of diabetic retinopathy, diabetic macular edema and related vision loss. Eye Vis 2015;2:17.

30. Zhou M, Astell-Burt T, Bi Y, et al. Geographical variation in diabetes prevalence and detection in china: multilevel spatial analysis of 98,058 adults. Diabetes Care 2015;38:72-81.

31. Liu Y, Song Y, Tao L, et al. Prevalence of diabeticretinopathy among 13473patients with diabetesmellitus in China: a cross sectionalepidemiologicalsurvey in six provinces. BMJOpen 2017;7:e013199.

32. Harding S, Greenwood R, Aldington S, et al. Grading and disease management in national screening for diabetic retinopathy in England and Wales. Diabet Med 2003:20:965-71.

33. Hypertension - diagnosis and management. 2015 http://www2.gov. bc.ca/gov/content/health/practitioner-professional-resources/bcguidelines/hypertension.

34. Mansfield ER, Helms BP. Detecting multicollinearity. Am Stat 1982;36:158-60.

35. Semeraro F, Parrinello G, Cancarini A, et al. Predicting the risk of diabetic retinopathy in type 2 diabetic patients. J Diabetes Complications 2011:25:292-7.

36. Nakamura M, Barber AJ, Antonetti DA, et al. Excessive hexosamines block the neuroprotective effect of insulin and induce apoptosis in retinal neurons. J Biol Chem 2001;276:43748-55.

37. Juutilainen A, Lehto S, Rönnemaa T, et al. Retinopathy predicts cardiovascular mortality in type 2 diabetic men and women. Diabetes Care 2007;30:292-9.

38. Fauziana R, Jeyagurunathan A, Abdin E, et al. Body mass index, waist-hip ratio and risk of chronic medical condition in the elderly population: results from the Well-being of the Singapore Elderly (WiSE) Study. BMC Geriatr 2016;16:125.

39. Man RE, Sabanayagam C, Chiang PP, et al. Differential Association of Generalized and abdominal obesity with diabetic retinopathy in Asian patients with Type 2 diabetes. JAMA Ophthalmol 2016;134:251-7.

40. Eriksson J, Jousilahti P, Lindström J, et al. Is fasting glucose sufficient to define diabetes? Epidemiological data from 20 European studies. Diabetologia 1999;42:647-54.

41. Hyvärinen M, Tuomilehto J, Mähönen M, et al. Hyperglycemia and incidence of ischemic and hemorrhagic stroke-comparison between fasting and 2-hour glucose criteria. Stroke 2009;40:1633-7.

42. Ning F, Tuomilehto J, Pyörälä K, et al. Cardiovascular disease mortality in Europeans in relation to fasting and 2-h plasma glucose levels within a normoglycemic range. Diabetes Care 2010;33:2211-6.

43. Guillausseau PJ, Meas T, Virally M, et al. Abnormalities in insulin secretion in type 2 diabetes mellitus. Diabetes Metab 2008;34(Suppl 2):S43-8. 